\title{
Can retrohepatic tunnel be quickly and easily established for laparoscopic liver hanging maneuver by Goldfinger dissector in laparoscopic right hepatectomy?*\#
}

\author{
Liu-xin $\mathrm{CAI}^{\S}$, Fang-qiang $\mathrm{WEI}^{\S}$, Yi-chen YU, Xiu-jun $\mathrm{CAI}^{\dagger+}$ \\ (Department of General Surgery, Institute of Minimally Invasive Surgery, Sir Run Run Shaw Hospital, School of Medicine, \\ Zhejiang University, Hangzhou 310016, China) \\ †E-mail: cxjzu@hotmail.com
}

Received Apr. 27, 2016; Revision accepted June 23, 2016; Crosschecked Aug. 20, 2016

\begin{abstract}
Objective: The liver hanging maneuver (LHM) is rarely applied in laparoscopic right hepatectomy (LRH) because of the difficulty encountered in retrohepatic tunnel (RT) dissection and tape positioning. Thus far no report has detailed how to quickly and easily establish RT for laparoscopic LHM in LRH, nor has employment of the Goldfinger dissector to create a total RT been reported. This study's aim was to evaluate the safety and feasibility of establishing RT for laparoscopic LHM using the Goldfinger dissector in LRH. Methods: Between March 2015 and July 2015, five consecutive patients underwent LRH via the caudal approach with laparoscopic LHM. A five-step strategy using the Goldfinger dissector to establish RT for laparoscopic LHM was adopted. Perioperative data were analyzed. Results: The median age of patients was 58 (range, 51-65) years. Surgery was performed for one intrahepatic lithiasis and four hepatocellular carcinomas with a median size of $90(40-150) \mathrm{mm}$. The median operative time was $320(282-358) \mathrm{min}$ with a median blood loss of 200 (200-600) ml. Laparoscopic LHM was achieved in a median of 31 (21-62) min, and the median postoperative hospital stay was 14 (9-16) d. No transfusion or conversion was required, and no severe liver-related morbidity or death was observed. Conclusions: The Goldfinger dissector is a useful instrument for the establishment of RT. A five-step strategy using the Goldfinger dissector can quickly and easily facilitate an RT for a laparoscopic LHM in LRH.
\end{abstract}

Key words: Retrohepatic tunnel, Liver hanging maneuver, Goldfinger dissector, Laparoscopic right hepatectomy http://dx.doi.org/10.1631/jzus.B1600180 CLC number: R605

\section{Introduction}

Laparoscopic hepatectomy has become widespread (Nguyen et al., 2009; Cai et al., 2014; Wakabayashi et al., 2015) among surgeons alongside the

\footnotetext{
ॠ Corresponding author

${ }^{\S}$ The two authors contributed equally to this work

* Project supported by the General Research Project of Medicine and Science of Zhejiang Province (Nos. 2014KYB1 19 and 2015KYB221), China

\# Electronic supplementary materials: The online version of this article (http://dx.doi.org/10.1631/jzus.B1600180) contains supplementary materials, which are available to authorized users

(DD ORCID: Liu-xin CAI, http://orcid.org/0000-0002-6734-5949

(C) Zhejiang University and Springer-Verlag Berlin Heidelberg 2016
}

advancement of laparoscopic devices and accumulated expertise in laparoscopic surgery. The clinical application of laparoscopic right hepatectomy (LRH) is also on the increase (Dagher et al., 2009; Han et al., 2010; Lainas et al., 2015). However, LRH still remains a challenging procedure owing to the technical difficulty (Pearce et al., 2011; Tzanis et al., 2013) of resecting the deepest and largest portion of the liver. Moreover, LRH is always considered risky, especially in patients with large tumors located in the right side of the liver, which is associated with potentially massive intraoperative bleeding and a high conversion rate. Open right hepatectomy (ORH) has always been preferred for these patients. 
In such patients the liver hanging maneuver (LHM), originally established by Belghiti et al. (2001), can overcome such technical difficulty and is always used in ORH via the anterior approach (Lai et al., 1996). Specifically, the technique consists of passing a hanging tape through a retrohepatic tunnel (RT) anterior to the inferior vena cava (IVC) and elevating the liver during liver parenchymal transection, thus facilitating the anterior approach and minimizing the risks of massive bleeding, iatrogenic tumor rupture, and tumor cell dissemination by avoiding liver mobilization (Wang et al., 2010; Wu et al., 2010; Beppu et al., 2012).

However, for patients with large right-sided liver tumors it remains largely unknown whether LHM can be used under laparoscopy to minimize the risk of massive bleeding and reduce the conversion rate for LRH while guaranteeing the safety of the procedure. In fact, laparoscopic LHM has not been commonly used in clinical practice because it is technically demanding and potentially hazardous. Its main challenge is difficulty in quickly and easily establishing an RT because of problems encountered in having to blindly perform RT dissection and tape positioning, the risk of potential vascular injury as also occurs in ORH, and, more importantly, the loss of tactile feedback under laparoscopy.

At present, reports on the effectiveness of laparoscopic LHM are scarce (Dokmak et al., 2014). In addition, no studies have provided details for others to learn about how to quickly and easily establish RT for laparoscopic LHM. With our extensive practice in laparoscopic hepatectomy (Cai et al., 2014; 2016) and experience with LHM in difficult open liver resections and LRH via the caudal approach, we found that for the caudal approach in the caudal-to-cranial direction, RT dissection offers some distinct characteristics and helps to overcome the aforementioned technical difficulties. Through innovative application of the Goldfinger dissector (initially designed for retrogastric tunnel dissection) in RT dissection, we have constructed a five-step strategy using this dissector to quickly and easily establish RT for laparoscopic LHM and facilitate the safe execution of LRH via the caudal approach.

The aim of this study was to evaluate the safety and feasibility of this novel technique.

\section{Materials and methods}

\subsection{Patients}

From June 2007 to July 2015, 40 LRHs were performed at our center (Department of General Surgery, Institute of Minimally Invasive Surgery, Sir Run Run Shaw Hospital, School of Medicine, Zhejiang University, Hangzhou, China). Ten of these patients underwent LRH via the caudal approach, five of whom consecutively underwent laparoscopic LHM between March 2015 and July 2015. A five-step strategy using the Goldfinger dissector was devised to quickly and easily establish RT for laparoscopic LHM in each patient.

\subsection{Surgical technique}

\subsubsection{Equipment, position, and trocar placement}

Generally, the basic laparoscopic equipment required was similar to that described for our previous reports of laparoscopic hepatectomy (Cai et al., 2014; 2016). The Goldfinger dissector (Blunt Dissectors, OBTECH Medical SARL, Johnson \& Johnson, Le Locle, Switzerland; Fig. 1) was mainly used for RT dissection and tape positioning. The laparoscopic multifunctional operative dissector (LMOD) (Cai et al., 2014 ; 2016), which is routinely used for laparoscopic liver transection in our center, was employed in all procedures. The patient was placed in the supine and $30^{\circ}$ reverse Trendelenburg position with elevation of

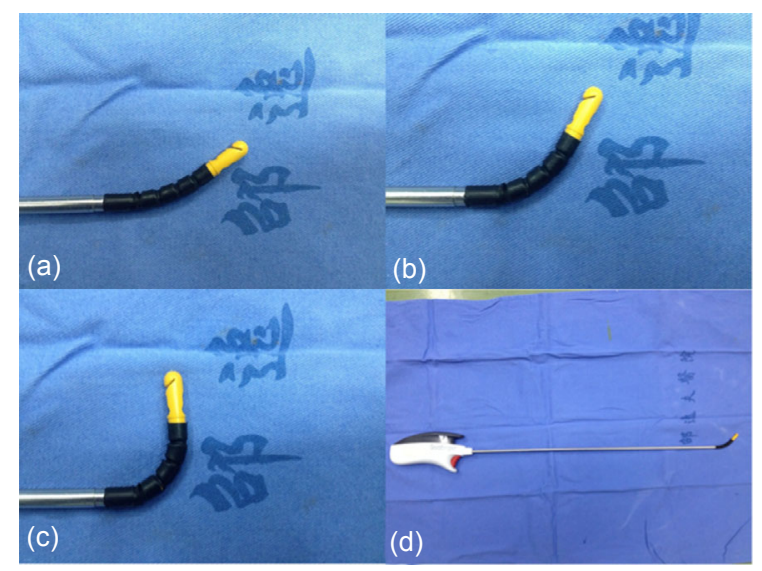

Fig. 1 Tip of the Goldfinger dissector with precise articulation, which can be flexing between $30^{\circ}$ (neutral position) and $90^{\circ}$

(a) $30^{\circ}$ (neutral position); (b) $45^{\circ}$; (c) $90^{\circ}$; (d) Overview of the Goldfinger dissector 
the right upper abdomen, with the principal surgeon standing on the patient's left side. Five ports were generally placed in the abdomen (Fig. 2). The first 10 -mm port (A) placed $1 \mathrm{~cm}$ below the umbilicus was used as the optical port. A 12-mm port (B) placed $1 \mathrm{~cm}$ below the xyphoid acted as the main manipulation port allowing the entry of the LMOD. Two assisting $5-\mathrm{mm}$ ports $(\mathrm{C}, \mathrm{D})$ were placed at the right midclavicular line and midaxillary line, respectively. The last 12-mm port (E) placed at mid-distance between port $\mathrm{A}$ and port $\mathrm{C}$ allowed entry of the Goldfinger dissector, specifically for RT dissection and tape positioning.

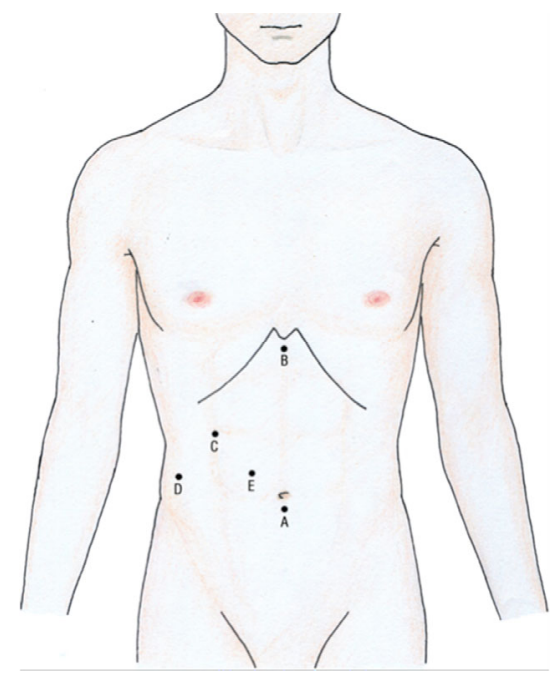

Fig. 2 Port sites for laparoscopic right hepatectomy

Port A: a $10-\mathrm{mm}$ port placed $1 \mathrm{~cm}$ below the umbilicus was used as the optical port; Port B: a 12-mm port placed $1 \mathrm{~cm}$ below the xyphoid acted as the main manipulation port allowing the entry of the laparoscopic multifunctional operative dissector (LMOD); Ports C and D: two assistant 5-mm ports were placed at the right midclavicular line and midaxillary line, respectively; Port E: this assistant $12-\mathrm{mm}$ port placed at mid-distance between port $\mathrm{A}$ and port $\mathrm{C}$ allowed the entry of the Goldfinger dissector for retrohepatic tunnel (RT) dissection and tape positioning

\subsubsection{Liver hilar dissection}

First, a cholecystectomy was performed but with the gallbladder left in situ for retraction. Second, by meticulous blunt dissection of the hepatoduodenal ligament, the right hepatic artery was visualized and then clamped with absorbable clips or Hem-o-loks (Teleflex Medical, Research Triangle Park, NC, USA). The portal trunk was then cautiously dissected until there was clear identification of the portal bifurcation and the right portal branch, which was then sutured or occluded with absorbable clips or Hem-o-loks. Next, the right portion of the hilar plate was accessed and lowered for safe dissection of the right hepatic duct, which was then completely separated from the liver parenchyma and also occluded with absorbable clips or Hem-o-loks before transection. A demarcation line could be identified after right selective vascular inflow occlusion was accomplished.

\subsubsection{Quick and easy establishment of RT}

After liver hilar dissection, a five-step strategy was implemented in the caudal-to-cranial direction. First, the peritoneum reflection anterior to the infrahepatic IVC was incised and dissected by the LMOD. The initial caudal portion of the RT (initial cad-RT) anterior to the IVC was developed (Fig. S1). If present and necessary, one to two branches of the short hepatic veins (SHVs) around the cad-RT were ligated with Hem-o-loks. Second, guided by the Goldfinger dissector, a 1- to 2-cm-deep transection of the liver parenchyma was performed along the confluence of the caudate process and paracaval portion anterior to the RT by the LMOD. The Goldfinger dissector was inserted through the tunnel for blunt dissection with precise articulation of its versatile tip flexing between $30^{\circ}$ and $45^{\circ}$, thus establishing the cad-RT (approximately one-third of the total RT), as shown in Fig. 3. Third, the ligamentum teres and the falciform ligament were divided until the second porta hepatis was clearly exposed. The fossa of the superior hepatic vein was exposed by dissecting the space between the right hepatic vein (RHV) and middle hepatic vein by the LMOD; at this fossa, the end of the cranial portion of the RT (cra-RT) was located. Similarly, the Goldfinger dissector was used for meticulous blunt dissection through this fossa in a cranial-to-caudal direction with its tip flexing between $30^{\circ}$ and $90^{\circ}$, thus developing the cra-RT (approximately one-third of the total RT), as shown in Fig. 4. Fourth, the Goldfinger dissector was advanced from the cad-RT in a caudal-to-cranial direction with its tip flexing at $30^{\circ}$, forming the median portion of the RT (med-RT) (approximately one-third of the total RT), as shown in Fig. S2. Fifth, the total RT was completed and connected by the Goldfinger dissector via the med-RT in a cranial direction with its tip flexing between neutral $30^{\circ}$ and $90^{\circ}$ (Fig. S3). 


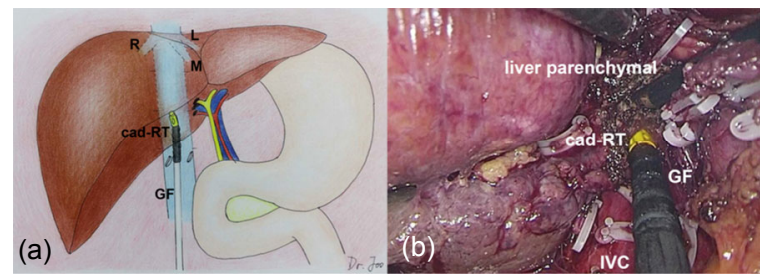

Fig. 3 Establishing the caudal portion of the retrohepatic tunnel (cad-RT)

Guided by the Goldfinger dissector (GF), 1- to 2-cm deep transection of the liver parenchymal was performed along the confluence of the caudate process and paracaval portion anterior to the RT by laparoscopic multifunctional operative dissector. The GF was inserted through the tunnel for blunt dissection with precise articulation of its versatile tip flexing between $30^{\circ}$ and $45^{\circ}$, establishing the cad-RT (approximately one-third of the total RT). R: right hepatic vein; M: middle hepatic vein; L: left hepatic vein; IVC: inferior vena cava. This step is described with illustration (a) and photograph (b), respectively

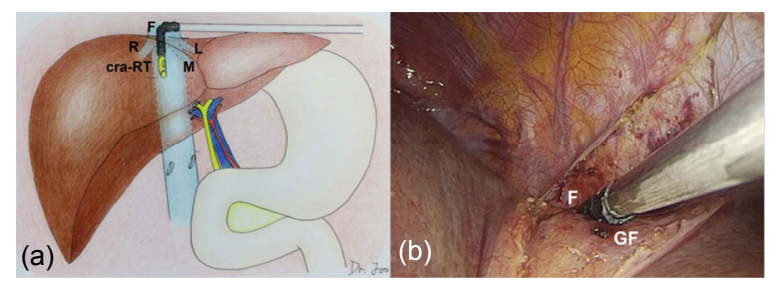

Fig. 4 Establishing the cranial portion of the retrohepatic tunnel (cra-RT)

The fossa of the superior hepatic vein $(\mathrm{F})$ was exposed by dissecting the space between the right hepatic vein $(R)$ and middle hepatic vein $(\mathrm{M})$ by the laparoscopic multifunctional operative dissector. The Goldfinger dissector (GF) was used for blunt dissection through this fossa in a cranial to caudal direction with its tip flexing between $30^{\circ}$ and $90^{\circ}$, thus developing the cra-RT (approximately one-third of the total RT). L: left hepatic vein. This step is described with illustration (a) and photograph (b), respectively

\subsubsection{Achievement of laparoscopic LHM}

After this five-step procedure, the Goldfinger dissector was inserted into the RT with its tip end visible through the fossa of the superior hepatic vein, and a 3-0 leading Vicryl (Johnson \& Johnson, New Brunswick, NJ, USA) was inserted into the chink of the tip end and then passed through the whole RT by the Goldfinger dissector (Fig. 5). The cranial end of the previous leading Vicryl was then secured to the end of a hanging tape (\#8 catheter) to form a loop, and the caudal end of the leading Vicryl was retracted from the cranial-to-caudal direction to follow the loop out of the whole RT. When the retaining suture of the loop was divided, the hanging tape was successfully and completely placed through the RT (Fig. S4).

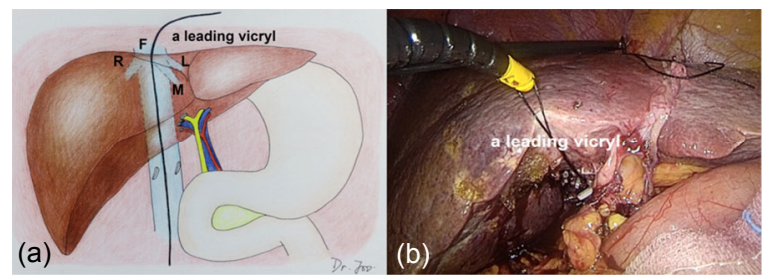

Fig. 5 A 3-0 leading Vicryl, which was passed through the whole retrohepatic tunnel (RT)

The Goldfinger dissector was inserted through the RT with its tip end visible through the fossa of the superior hepatic vein (F), and a 3-0 leading Vicryl was inserted into the chink of the tip end and then passed through the whole RT by the Goldfinger dissector. R: right hepatic vein; $\mathrm{M}$ : middle hepatic vein; L: left hepatic vein. This step is described with illustration (a) and photograph (b), respectively

\subsubsection{Parenchymal transection by laparoscopic LHM}

After achievement of laparoscopic LHM, the transection line was even more accurate with the guide from the tape. The hanging tape was then tensioned and banded along the Cantlie's line, and the anterior inferior part of the median hepatic fissure was first transected using the LMOD and the technique of curettage and aspiration (Cai et al., 2014; 2016) to the right of this guiding tape (Fig. 6). The tape was then loosened, and the assistant used grasping forceps to seize the cranial part of the tape tightly while the caudal part of the tape was retracted from port $\mathrm{E}$ to trigger lifting tension. With this lifting tension effect, the posterior superior part of the median hepatic fissure was transected (Fig. S5).

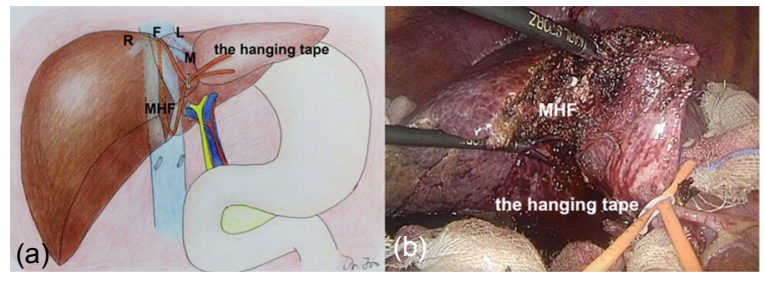

Fig. 6 Parenchymal transection of the anterior inferior part of the median hepatic fissure

The hanging tape was tensioned and banded along the Cantlie's line, and the anterior inferior part of the median hepatic fissure (MHF) was first transected using the LMOD and the technique of curettage and aspiration to the right of this guiding tape. R: right hepatic vein; M: middle hepatic vein; L: left hepatic vein; F: fossa of the superior hepatic vein. This step is described with illustration (a) and photograph (b), respectively 


\subsubsection{Ligation of SHVs and RHV}

Next, the anterior surface of the IVC was clearly exposed. Each branch of the SHVs was ligated individually, and the RHV was dissected and ligated intrahepatically by silk suture or an Endo-GIA stapler.

\subsubsection{Mobilization of the right part of the liver}

Proper hemostasis was required to ensure good control of bleeding from the deep parenchyma when we transected the parenchyma. The right part of the liver was then mobilized out of the retroperitoneum after the hepatorenal, right coronary, and triangular ligaments were divided.

\subsubsection{Hemostasis and specimen removal}

After mobilization of the liver, care was taken to examine for any suspected bleeding and bile leakage on the raw transected surface of the liver (Fig. S6). Minor bleeding and small bile strictures were immediately coagulated. Large vessels and bile ducts were sutured or clamped by absorbable clips or Hem-o-loks. The specimen was then removed through a remote suprapubic incision. A drainage tube was placed in the event of abdominal fluid collection.

\subsection{Study parameters}

Parameters comprised operating time, blood loss, use of IVC exclusion, blood transfusion, conversion rate, tumor characteristics, surgical margin, mortality, postoperative complications, readmissions, reoperation and postoperative hospital stay, follow-up time, and survival outcomes. Complications were graded according to the Clavien-Dindo system (Clavien et al., 2009). Categorical data were expressed as numbers (percentages) and continuous data as medians (with ranges). All data were entered and analyzed using SPSS Version 21.0 (SPSS, Chicago, IL, USA).

\section{Results}

\subsection{Preoperative characteristics}

From June 2007 to July 2015, 40 patients underwent LRH, five of whom received laparoscopic LHM in combination with the caudal approach using the Goldfinger dissector. Four males and one female (aged from 51 to 65 years, median age 58 years) were enrolled in the study. Four were diagnosed with hepatocellular carcinoma (HCC) and one with intrahepatic lithiasis. Details of patient demographics and clinical data are listed in Table 1.

Table 1 Patient demographics and clinical data $(n=5)$

\begin{tabular}{lc}
\hline \multicolumn{1}{c}{ Characteristics } & Value \\
\hline Age (year) & $58(51-65)$ \\
Male sex & $4(80 \%)$ \\
BMI $\left(\mathrm{kg} / \mathrm{m}^{2}\right)$ & $24.0(18.4-30.7)$ \\
Diagnosis & \\
$\quad$ HCC & $4(80 \%)$ \\
$\quad$ Intrahepatic lithiasis & $1(20 \%)$ \\
ECOG performance status & \\
$\quad 0 / 1$ & $5(20 \%) / 4(80 \%)$ \\
ASA score, II & $2(40 \%)$ \\
HBsAg positive & $2(40 \%)$ \\
Cirrhosis & $13.7(12.6-15.0)$ \\
Haemoglobin $(\mathrm{g} / \mathrm{dl})$ & $13.5(12.9-13.9)$ \\
Prothrombin time $(\mathrm{s})$ & $15.0(7.2-34.1)$ \\
Total bilirubin $(\mu \mathrm{mol} / \mathrm{L})$ & $34(27-79)$ \\
AST $(\mathrm{U} / \mathrm{L})$ & $52(28-88)$ \\
ALT $(\mathrm{U} / \mathrm{L})$ &
\end{tabular}

Values are expressed as median (range) or number (percentage). BMI: body mass index; HCC: hepatocellular carcinoma; ECOG: eastern cooperative oncology group; ASA: American Society of Anesthesiologists; HBsAg: hepatitis B surface antigen; AST: aspartate aminotransferase; ALT: alamine aminotransferase

\subsection{Intraoperative data}

Intraoperative data are shown in Table 2. Our technique was successful in all five patients. No procedures required conversions. The median operative time was 320 (range, 282-358) min with median blood loss of 200 (range, 200-600) ml. No catastrophic intraoperative bleeding occurred and no transfusion was required. The median time for achievement of laparoscopic LHM, parenchymal transection, RHV ligation, and right liver mobilization was 31 (range, 21-62), 132 (110-144), 11 (6-12), and 18 (6-25) min, respectively. Among the four HCC patients, the median maximum tumor diameter, tumor number, and surgical margin were 90 (range, 40-150) mm, 1 (1-2), and 17.5 (10-20) mm, respectively.

\subsection{Postoperative outcomes}

Postoperative outcomes are summarized in Table 3. The median postoperative hospital stay was 
14 (range, 9-16) d, and the median follow-up time for the four HCC patients was 10 (range, 7-11) months. All four of these HCC patients are alive without tumor recurrence. We observed no liver-related morbidity or death, although two postoperative complications occurred. One patient had postoperative pleural effusion (grade II), which was managed without surgical intervention. Another patient who had undergone intraoperative IVC exclusion developed postoperative renal insufficiency (grade IVa). Immediate multidisciplinary team support and continuous renal replacement therapy were initiated for this patient, who recovered within 2 weeks postoperatively.

Table 2 Intraoperative data $(n=5)$

\begin{tabular}{lc}
\hline \multicolumn{1}{c}{ Characteristics } & Value \\
\hline Operating time (min) & $320(282-358)$ \\
Total & $31(21-62)$ \\
Laparoscopic LHM & $132(110-144)$ \\
Parenchymal transection & $11(6-12)$ \\
RHV ligation & $18(6-25)$ \\
Right liver mobilization & $200(200-600)$ \\
Blood loss (ml) & $1(20 \%)$ \\
Patients requiring IVC exclusion & ${ }^{*}$ \\
$\quad$ Time for IVC exclusion (min) & 50 \\
Maximum diameter of malignant & $90(40-150)$ \\
$\quad$ lesions (mm) & $1(1-2)$ \\
$\begin{array}{l}\text { Number of malignant lesions } \\
\text { Surgical margin for malignant } \\
\text { lesions (mm) }\end{array}$ & $17.5(10-20)$ \\
\hline $\begin{array}{l}\text { Values are expressed as median (range). } \\
\text { LHM: Number (percentage). } \\
\text { inferior vena cava }\end{array}$ &
\end{tabular}

Table 3 Postoperative outcomes $(n=5)$

\begin{tabular}{lc}
\hline \multicolumn{1}{c}{ Characteristics } & Value \\
\hline Overall complications & $2(40 \%)$ \\
Clavien-Dindo grading ${ }^{*}$ & \\
II & 1 (pleural effusion) \\
IVa & 1 (renal dysfunction, \\
& requiring CRRT) \\
Readmission, $n$ & $0(0 \%)$ \\
Reoperation, $n$ & $0(0 \%)$ \\
Hospital stay (d) & $14(9-16)$ \\
Mortality, $n$ & $0(0 \%)$ \\
\hline
\end{tabular}

Values are expressed as median (range) or number (percentage). ${ }^{*}$ Words in parentheses are specific explanation. CRRT: continuous renal replacement therapy

\section{Discussion}

In ORH, the successful achievement of the original LHM technique depends on RT dissection, which remains the most technically challenging step of this procedure (Belghiti et al., 2001). Wu et al. (2010) successfully performed 33 ORHs via the anterior approach with LHM. They used a vascular clamp for RT dissection and placed a drainage tube through the RT to suspend the liver away from the retrohepatic IVC. However, laparoscopic LHM is more technically difficult because of restricted manipulation space available for blind RT dissection and tape positioning, as well as absolute lack of guidance of surgeons' fingers under laparoscopy.

Although a few surgeons have reported the use of laparoscopic LHM, none of the studies to date has reported how to perform the procedure in detail. Dokmak et al. (2014) demonstrated their experience with the use of laparoscopic LHM in five pure LRHs, and provided a video showing that laparoscopic LHM was achieved via the up-to-down direction using a nasogastric tube for tunneling in a 54-year-old female patient with large telangiectatic hepatocellular adenomas $(>5 \mathrm{~cm})$. However, surgeons cannot directly observe the RT from the up-to-down direction under laparoscopy and thus have a greatly obstructed view of manipulation, while the nasogastric tube is soft and negotiates the RT with difficulty. Moreover, this report lacks a clear and detailed explanation of RT dissection, which would be of benefit to other surgeons wishing to learn and perform the procedures.

Considering the great challenge of laparoscopic LHM, some clinicians have attempted modifications to LHM in LRH. Instead of establishing an RT, Kim et al. (2016) recently proposed a modification of LHM in LRH by placing a hanging tape through a different avascular plane after liver mobilization. The upper end of the tape was located at the lateral side of the RHV while the lower end was positioned between the right and left Glisson pedicles. However, several drawbacks were associated with this newly modified technique. First, liver mobilization prior to parenchymal transection was risky under laparoscopy, especially in patients with huge tumors. Major concerns were massive bleeding caused by tumor rupture in the course of mobilization as well as tumor dissemination. Second, the RHV and SHVs were not 
well controlled, which may have contributed to excessive bleeding from the deep liver parenchyma. Moreover, the hanging tape that was placed along the lateral side of the IVC could not provide an accurate guide for the transection plane, especially when transecting the median hepatic fissure.

Throughout these reports, the greatest difficulty in achieving the original LHM is the inability to quickly and easily establish RT under laparoscopy. In this study, we adopted a totally laparoscopic LHM for all five patients. We innovatively applied the Goldfinger dissector for RT dissection in a laparoscopic LHM for LRH, which we consider an improvement over the vascular clamp (Wu et al., 2010) that cannot be used under laparoscopy and the soft nasogastric tube with poor guidance used by Dokmak et al. (2014) in LRH for LHM. Furthermore, a clear and detailed five-step strategy was adopted for laparoscopic LHM without liver mobilization, and a caudal-to-cranial direction was adopted for the procedure, also at variance with previous studies (Dokmak et al., 2014; Kim et al., 2016).

Currently there are no results available from large series regarding the use of laparoscopic LHM, nor have clear indications and contraindications been reported. As in ORH, the main indication for laparoscopic LHM is a large tumor invading the diaphragm or the right liver, which is thus difficult to mobilize because of tumor infiltration. The contraindication for laparoscopic LHM is a tumor invading the retrohepatic space anterior to the IVC and severe adhesions between the liver and the IVC. It was reported (Qin et al., 2012) that tumors larger than $13 \mathrm{~cm}$ at segments VI-VIII were not suitable for RT dissection. However, in our procedures all of the five patients, including two with large HCCs (11 and $15 \mathrm{~cm}$ in size), underwent RT dissection and laparoscopic LHM without conversions or manual assistance. Moreover, one patient with intrahepatic lithiasis also successfully underwent laparoscopic LHM.

As in ORH, a key to laparoscopic LHM was the establishment of the RT. The RT was meticulously dissected by the Goldfinger dissector, and a hanging tape was then passed through the RT to elevate the liver and achieve laparoscopic LHM. Liver parenchymal transection was then performed by LHM via the caudal approach. Our five-step strategy helped us to quickly and easily establish RT, which accounted for the successful achievement of tape positioning without massive intraoperative bleeding. Each step of the five-step strategy has its operational essentials and purpose. The achievement of laparoscopic LHM costs only a median of 31 (range, 21-62) min. We believe that adherence to each step of the five-step strategy leads to safe and effective achievement of RT dissection and laparoscopic LHM.

Another key factor in quickly and easily achieving laparoscopic LHM is the use of the Goldfinger dissector, which has an angled shape with its tip being capable of flexing between $30^{\circ}$ and $90^{\circ}$ (Figs. 1a, 1b, and 1c). This device was initially designed for placement of a gastric band through a retrogastric tunnel in laparoscopic bariatric surgery (Ceelen et al., 2003). Inspired by this, we applied the Goldfinger dissector in RT dissection, an approach that to our knowledge has not been reported to date. In our experience the Goldfinger dissector proffers several advantages. First, it has a moderate diameter and adequate length, fits well, and is able to cross the whole RT (Fig. 1). Second, it provides precise articulation with a versatile tip, and is useful in exposing the fossa of the superior hepatic vein and dissecting the space between the RHV and middle hepatic vein. Once its distal tip is passed through the fossa, the tape can be captured by its tip (Fig. 1) and then delivered smoothly along the tunnel with ease. In our series the Goldfinger dissector provided meticulous blunt dissection in tunneling and allowed us to avoid laceration of SHVs; its atraumatic and flexible tip (Fig. 1) could turn naturally along the 1-cm-wide RT when mild resistance was encountered, thus avoiding massive bleeding.

Bleeding is a major concern during hepatectomy and also is an important factor affecting the prognosis. In the initial experience of Belghiti et al. (2001), minor bleeding ceased spontaneously after compression of the parenchyma. If massive bleeding was encountered, portal triad clamping or IVC exclusion was suggested for its control (Wu et al., 2010). We reviewed reports of large series (of at least 10 cases) utilizing LRH with (Kim et al., 2016) or without LHM (Gayet et al., 2007; Dagher et al., 2008; Medbery et al., 2014; Lainas et al., 2015) and ORH with LHM (Wu et al., 2010) in recent years (Table 4). Our series presented a lower intraoperative blood loss in comparison with previous series of LRH with or without LHM, and ORH with LHM. Moreover, no patients required transfusions in our series. 
Table 4 Comparison with LRH or ORH from recent literatures

\begin{tabular}{lcccccccc}
\hline \multicolumn{1}{c}{ Author } & Year & Type & $\begin{array}{c}\text { Use of } \\
\text { LHM }\end{array}$ & $\begin{array}{c}\text { Number of } \\
\text { patients }\end{array}$ & $\begin{array}{c}\text { Operating time } \\
(\mathrm{min})^{\mathrm{a}}\end{array}$ & $\begin{array}{c}\text { Blood loss } \\
(\mathrm{ml})^{\mathrm{a}}\end{array}$ & $\begin{array}{c}\text { Conversion, } \\
n^{\mathrm{b}}\end{array}$ & $\begin{array}{c}\text { Mortality, } \\
n^{\mathrm{b}}\end{array}$ \\
\hline Gayet et al. & 2007 & LRH & No & 42 & $360(185-505)$ & NA & $6(14 \%)$ & $1(2.3 \%)$ \\
Dagher et al. & 2008 & LRH & No & 20 & $388 \pm 104$ & $563 \pm 523$ & $2(10 \%)$ & 0 \\
Medbery et al. & 2014 & LRH & No & 48 & $233 \pm 32$ & $281 \pm 306$ & $5(10.4 \%)$ & $2(4.2 \%)$ \\
Lainas et al. & 2015 & LRH & No & 45 & $272.3 \pm 12.4$ & $480.7 \pm 92.6$ & $5(11.1 \%)$ & 0 \\
Kim et al. & 2016 & LRH & Yes & 10 & $275(260-390)$ & $375(150-600)$ & 0 & 0 \\
Our series & 2016 & LRH & Yes & 5 & $320(282-358)$ & $200(200-600)$ & 0 & 0 \\
Wu et al. & 2010 & ORH & Yes & 33 & $373(283-478)$ & $500(300-725)$ & NA & $2(6.1 \%)$ \\
\hline
\end{tabular}

${ }^{a}$ Values are expressed as median (range) or mean \pm standard deviation; ${ }^{b}$ Values in parentheses are percentages unless indicated otherwise. LRH: laparoscopic right hepatectomy; ORH: open right hepatectomy; LHM: liver hanging maneuver; NA: not available

We attribute this to several factors such as the use of the LHM, the caudal approach, and effective vascular occlusion. First, the LHM provided protection to major vascular structures by lifting the liver, exposing sufficient space for dissection, and preventing the transection line from deviating from Cantlie's line In addition, holding two ends of a hanging tape together can minimize the risk of bleeding from venous backflow and thus facilitate bloodless transection. Second, the caudal approach is commensurate with the LHM in preventing unnecessary bleeding. Taking the caudal approach, the liver is mobilized at the completion of liver parenchymal transection, which helps to prevent potential bleeding caused by tumor rupture. Moreover, as the transection took place via the caudal-to-cranial route instead of the anteriorto-posterior approach of open surgery, the dorsal part of the liver could be better visualized and unnecessary injuries to the IVC or RHV avoided. Third, selective inflow occlusion was performed in all procedures. Specifically, the right hepatic artery and right portal vein were divided and clipped before parenchymal transection.

Besides less blood loss, our series showed a lower conversion rate ( $0 \%$ versus ranging from $10 \%$ to $14 \%$ ) compared with previous reports of LRH without LHM (Gayet et al., 2007; Dagher et al., 2008; Medbery et al., 2014; Lainas et al., 2015), and a less operating time compared with ORH with LHM (Wu et al., 2010). Operative time and mortality in our study were comparable with reports of previous LRH with (Kim et al., 2016) or without LHM (Gayet et al., 2007; Dagher et al., 2008; Medbery et al., 2014; Lainas et al., 2015), as shown in Table 4. Specifically, there was no severe liver-related morbidity, reoperation, readmission, or death in our series, demonstrating that our technique represents a safe and feasible approach. No recurrence was observed in four HCC patients after a median follow-up of 10 months. This may be a result of the radical resection of malignant lesions (a median surgical margin of $17.5 \mathrm{~mm}$ was obtained in HCC patients) and the use of our "no-touch" technique.

\section{Conclusions}

In conclusion, the Goldfinger dissector is a useful instrument for the establishment of RT. A five-step strategy involving use of the Goldfinger dissector helps to quickly and easily establish RT for laparoscopic LHM, and laparoscopic LHM facilitates LRH. This technique seems to be a safe and feasible approach. Admittedly, LRH is among the most challenging laparoscopic procedures and patients eligible for LRH are few in number. Therefore, the opportunity of applying this technique is limited. Nevertheless, more experience in a larger number of patients together with a randomized design is required to further delineate the benefits of this technique in future procedures.

\section{Acknowledgements}

We gratefully thank Dr. Quan-chao ZHU (Department of Plastic Surgery, Sir Run Run Shaw Hospital, School of Medicine, Zhejiang University, Hangzhou, China) for her excellent work in designing and drawing pictures particularly for us, and also thank Dr. Tu-nan YU (Department of General Surgery, Sir Run Run Shaw Hospital, School of Medicine, Zhejiang University, Hangzhou, China) for his warm help in the realization of the manuscript. 


\section{Compliance with ethics guidelines}

Liu-xin CAI, Fang-qiang WEI, Yi-chen YU, and Xiu-jun CAI declare that they have no conflict of interest.

All procedures followed were in accordance with the ethical standards of the responsible committee on human experimentation (institutional and national) and with the Helsinki Declaration of 1975, as revised in 2008 (5). Informed consent was obtained from all patients for being included in the study.

\section{References}

Belghiti, J., Guevara, O.A., Noun, R., et al., 2001. Liver hanging maneuver: a safe approach to right hepatectomy without liver mobilization. J. Am. Coll. Surg., 193(1): 109-111. http://dx.doi.org/10.1016/S1072-7515(01)00909-7

Beppu, T., Ishiko, T., Chikamoto, A., et al., 2012. Liver hanging maneuver decreases blood loss and operative time in a right-side hepatectomy. Hepatogastroenterology, 59(114):542-545. http://dx.doi.org/10.5754/hge09737

Cai, X.J., Li, Z.Y., Zhang, Y.L., et al., 2014. Laparoscopic liver resection and the learning curve: a 14-year, singlecenter experience. Surg. Endosc., 28(4):1334-1341. http://dx.doi.org/10.1007/s00464-013-3333-5

Cai, X.J., Duan, L., Wang, Y.F., et al., 2016. Laparoscopic hepatectomy by curettage and aspiration: a report of 855 cases. Surg. Endosc., 30(7):2904-2913. http://dx.doi.org/10.1007/s00464-015-4576-0

Ceelen, W., Walder, J., Cardon, A., et al., 2003. Surgical treatment of severe obesity with a low-pressure adjustable gastric band: experimental data and clinical results in 625 patients. Ann. Surg., 237(1):10-16.

Clavien, P.A., Barkun, J., de Oliveira, M.L., et al., 2009. The Clavien-Dindo classification of surgical complications: five-year experience. Ann. Surg., 250(2):187-196. http://dx.doi.org/10.1097/SLA.0b013e3181b13ca2

Dagher, I., Caillard, C., Proske, J.M., et al., 2008. Laparoscopic right hepatectomy: original technique and results. J. Am. Coll. Surg., 206(4):756-760. http://dx.doi.org/10.1016/j.jamcollsurg.2007.09.012

Dagher, I., di Giuro, G.J., Dubrez, J., et al., 2009. Laparoscopic versus open right hepatectomy: a comparative study. Am. J. Surg., 198(2):173-177. http://dx.doi.org/10.1016/j.amjsurg.2008.09.015

Dokmak, S., Ben Safta, Y., Ftériche, F.S., et al., 2014. Pure laparoscopic right hepatectomy with the hanging maneuver for multiple hepatocellular adenomas. Ann. Surg. Oncol., 21(12):3800-3801. http://dx.doi.org/10.1245/s10434-014-3809-0

Gayet, B., Cavaliere, D., Vibert, E., et al., 2007. Totally laparoscopic right hepatectomy. Am. J. Surg., 194(5):685-689. http://dx.doi.org/10.1016/j.amjsurg.2006.11.044

Han, H.S., Yoon, Y.S., Cho, J.Y., et al., 2010. Laparoscopic right hemihepatectomy for hepatocellular carcinoma. Ann.
Surg. Oncol., 17(8):2090-2091.

http://dx.doi.org/10.1245/s10434-010-1066-4

Kim, J.H., Ryu, D.H., Jang, L.C., et al., 2016. Lateral approach liver hanging maneuver in laparoscopic anatomical liver resections. Surg. Endosc., 30(8):3611-3617.

http://dx.doi.org/10.1007/s00464-015-4663-2

Lai, E.C.S., Fan, S.T., Lo, C.M., et al., 1996. Anterior approach for difficult major right hepatectomy. World $J$. Surg., 20(3):314-318. http://dx.doi.org/10.1007/s002689900050

Lainas, P., Camerlo, A., Conrad, C., et al., 2015. Laparoscopic right hepatectomy combined with partial diaphragmatic resection for colorectal liver metastases: is it feasible and reasonable? Surgery, 158(1):128-134. http://dx.doi.org/10.1016/j.surg.2015.02.003

Medbery, R.L., Chadid, T.S., Sweeney, J.F., et al., 2014. Laparoscopic vs open right hepatectomy: a value-based analysis. J. Am. Coll. Surg., 218(5):929-939. http://dx.doi.org/10.1016/j.jamcollsurg.2014.01.045

Nguyen, K.T., Gamblin, T.C., Geller, D.A., 2009. World review of laparoscopic liver resection-2804 patients. Ann. Surg., 250(5):831-841. http://dx.doi.org/10.1097/SLA.0b013e3181b0c4df

Pearce, N.W., di Fabio, F., Teng, M.J., et al., 2011. Laparoscopic right hepatectomy: a challenging, but feasible, safe and efficient procedure. Am. J. Surg., 202(5):e52-e58. http://dx.doi.org/10.1016/j.amjsurg.2010.08.032

Qin, Q., Wang, L., Li, H., et al., 2012. Efficacy and safety of right hemihepatectomy through the right retrohepatic tunnel. Cell Biochem. Biophys., 62(1):113-118. http://dx.doi.org/10.1007/s12013-011-9268-3

Tzanis, D., Shivathirthan, N., Laurent, A., et al., 2013. European experience of laparoscopic major hepatectomy. $J$. Hepatobiliary Pancreat. Sci., 20(2):120-124. http://dx.doi.org/10.1007/s00534-012-0554-2

Wakabayashi, G., Cherqui, D., Geller, D.A., et al., 2015. Recommendations for laparoscopic liver resection: a report from the second international consensus conference held in Morioka. Ann. Surg., 261(4):619-629. http://dx.doi.org/10.1097/SLA.0000000000001184

Wang, C.C., Jawade, K., Yap, A.Q., et al., 2010. Resection of large hepatocellular carcinoma using the combination of liver hanging maneuver and anterior approach. World $J$. Surg., 34(8):1874-1878. http://dx.doi.org/10.1007/s00268-010-0546-9

Wu, T.J., Wang, F., Lin, Y.S., et al., 2010. Right hepatectomy by the anterior method with liver hanging versus conventional approach for large hepatocellular carcinomas. Br. J. Surg., 97(7):1070-1078. http://dx.doi.org/10.1002/bjs. 7083

\section{List of electronic supplementary materials}

Fig. S1 Establishing the initial caudal portion of the retrohepatic tunnel (initial cad-RT) 
Fig. S2 Establishing the median portion of the retrohepatic tunnel (med-RT)

Fig. S3 Establishing the total retrohepatic tunnel (RT)

Fig. S4 Placement of the hanging tape through the whole retrohepatic tunnel (RT)

Fig. S5 Parenchymal transection of the median hepatic fissure (MHF)

Fig. S6 Achievement of hemostasis after removal of the specimen

\section{中文概要}

\section{题 目: 能否运用金手指在腹腔镜右半肝切除术中快速简 易建立肝后隧道实行绕肝带提拉?}

目 的：评估金手指在腹腔镜右半肝切除术中快速简易建 立肝后隧道实行绕肝带提拉的可行性和安全性。

创新点: 在腹腔镜右半肝中很少采用绕肝带提拉, 这是因 为在腹腔镜下解剖肝后隧道和置放绕肝带难度 极大。本文首次详细报道在腹腔镜右半肝切除术 中快速简易建立肝后隧道来实行绕肝带提拉，同
时首次报道运用金手指在腹腔镜下建立完整的 肝后隧道。

方 法: 选取 2015 年 3 月至 7 月间 5 例连续接受前入路腹 腔镜右半肝切除术并行绕肝带提拉的患者为研 究对象, 运用金手指在腹腔镜下进行 5 步法建立 肝后隧道, 收集和研究这些患者的手术资料。

结 论: 这 5 例患者的中位年龄为 58 岁 (范围 51 65 岁)。 其中, 1 例患有肝内胆管结石, 4 例患有肝细胞性 肝癌。肿瘤中位直径为 $90 \mathrm{~mm}$ (范围 $40 \sim 150 \mathrm{~mm}$ ), 手术中位时间为 $320 \mathrm{~min}$ （范围 282 358 min）, 中位失血量为 $200 \mathrm{ml}$ (范围 200 600 ml), 腹腔 镜下绕肝带提拉中位完成时间为 $31 \mathrm{~min}$ (范围 21 62 min), 术后中位住院时间为 $14 \mathrm{~d}$ (范围 9 16 d)。术中未行输血, 未发生中转开腹; 术 后并未发生严重肝脏并发症, 无患者死亡。因此 运用金手指进行肝后隧道建立是有效可行的。采 取 5 步法可快速简易建立肝后隧道, 有助于在腹 腔镜右半肝切除术中安全有效进行绕肝带提拉。

关键词: 肝后隧道; 绕肝带提拉; 金手指; 腹腔镜右半肝 切除术 